\title{
Pulsion de destruction et mort en acte. Clinique du sujet en guerre
}

\author{
Paul-Laurent Assoun
}

« Heureusement que la guerre est si terrible, autrement nous finirions par trop l'aimer. »

Ce propos n'émane pas de quelque paradoxe analytique, mais de l'aveu d'un homme de guerre ${ }^{1}$, qui vaut son pesant d'or pour introduire l'opération inconsciente en cause. Comment le sujet peut-il s'avérer jouir de cela même qui le terrifie et le menace de destruction, au point qu'il doive s'en rappeler à lui-même le caractère terrifiant, pour s'avertir de résister à cela que l'on peut appeler... « l'amour de la guerre » ? Réintroduction à la question psychanalytique : «Pourquoi de la guerre ?» Telle est la lettre de l'interrogation freudienne (Warum Krieg ?), plus exacte que «Pourquoi la guerre ? » En cette dernière interrogation, « la guerre » est présentée, selon un procédé inauguré par la Psychomachie $^{2}$, comme un principe allégorique. Il faut faire chuter le

Paul-Laurent Assoun, psychanalyste, professeur à l'Université Paris-Diderot Paris 7, directeur de recherche membre du Centre de recherche psychanalyse, médecine et société (CRPMS).

1. Propos attribué à Robert E. Lee, général sudiste pendant la guerre de Sécession américaine, cité par Jesse Glenn Gray, Au combat. Réflexions sur les hommes à la guerre (The Warriors. Reflections on Men in Battle, 1959, Paris, Tallandier, 2012). Sur le contexte, voir infra.

2. Lucain, Psychomachia, poème du poète latin de la fin du $\mathrm{III}^{\mathrm{e}}$ siècle réputé introduire le mode de pensée allégorique, à travers le « combat de l'âme ». 
substantif pour mieux entendre le partitif. Telle est en ce sens la question freudienne, dont hérite la psychanalyse. L'éventualité anthropologique chronique de la guerre n'est pas un objet mineur en psychanalyse, ni un simple propos de circonstance - fût-ce celle, massive, de la première guerre de masse, qui a convoqué Freud à prendre la plume sur la guerre et la mort, inséparables. C'est de plus pour le créateur de la psychanalyse un phénomène révélateur à la fois d'une figure majeure du politique - la guerre étant depuis Clausewitz caractérisée comme « la continuation de la politique par d'autres moyens ${ }^{3} \gg-$ et de la fonction inconsciente de l'agressivité. La guerre est bien la question du politique, son moment de vérité. Le réexamen de la position freudienne sur la question s'avère précieux pour l'actualité même - actualité chronique en quelque sorte - du phénomène guerrier.

\section{POURQUOI LA GUERRE ? ENJEUX INCONSCIENTS D'UN DRAME}

Or, chez Freud, il y a un croisement intime entre :

- d'une part, un discours contextuel à l'actualité pressante et oppressante, lié au conflit mondial et à la nécessité pour Freud de prendre position, pendant la Première Guerre dite mondiale, mais aussi dans le malaise nommé « entre-deux-guerres », ce qu'attestent respectivement les écrits de 1915 - sommet de sa réflexion - et de 1932, année de la correspondance avec Einstein, «lettre ouverte » publiée sous l'égide de la Société des nations ${ }^{4}$. Voilà qui marque la prise de la psychanalyse dans le discours de (sur) la guerre : qu'a-t-elle à énoncer en propre sur la guerre, d'un discours, assurément, mais qui ne soit « du semblant », dès lors que l'on a affaire au réel même du collectif ? Discours par définition «en contexte », car c'est le contexte qui convoque à (la) penser, sauf à démasquer à un certain moment le contextuel

3. C. von Clausevitz, De la guerre, 1832.

4. Nous renvoyons à notre commentaire de ce texte dans «Correspondance Freud-Einstein », Hermes $n^{\circ}$ 5/6, juillet-septembre 1982, p. 261-273 et à l'étude « Freudisme et indifférentisme politique », Hermes n ${ }^{\circ}$ 5/6, p. 345-359. Sur la présentation de la genèse du contenu et des enjeux de ce texte-clé, voir l'article que nous lui avons consacré dans notre Dictionnaire psychanalytique des cuvres psychanalytiques, Paris, Puf, 2009, p. 956-961. 
pour dégager textuellement cette « signification hors contexte » qu'est le réel inconscient ;

- d'autre part, une question clinique et métapsychologique, celle qui mène à la question du trauma - dont elle renouvelle la clinique, sinon la problématique -, et de la mort, dans le fairecuvre de mort collectif, ce qui convoque une seconde fois au réel. Car l'on n'est peut-être jamais plus ensemble que dans l'élément séparateur de la guerre.

Cela dessine l'articulation entre les deux dimensions du sujet de la guerre, sur le plan du statut de l'agressivité et dans son rapport à la Culture.

\section{Guerre, État et Histoire}

Le mot « guerre », qui a commencé par désigner des troubles, un désordre, un scandale, une querelle, s'est spécifié très tôt dans l'acception de « lutte armée entre groupes humains ou entre États ». Elle suppose une dissension entre particuliers ou entre entités étatiques. Elle implique donc la notion d'ennemi et l'idée d'une confrontation durable à cet « ennemi » (c'est le surnom du Diable dans l'usage chrétien primitif). Elle est ainsi corrélative de la notion d'État, qui doit être constitué pour entrer en guerre avec ses pairs, les autres États. Point de guerre sans jalousie entre États, les «causes » de guerre étant ce qui nourrit le brasier de ce zèle agressif, jusqu'en ce point où elle ne peut plus se dénouer que dans et par l'affrontement.

La guerre n'est pas qu'un événement de l'Histoire, elle définit et nomme une tranche d'histoire : on parle de «l'avantguerre » et de «l'entre-deux-guerres », en sorte qu'elle découpe diachroniquement le devenir historique. Signe de son incidence traumatique, elle fait incise dans la continuité de l'histoire, inscrivant la coupure irrésorbable entre un « avant » et un « après » (elle). Bref, la Guerre s'incarne comme envers du lien social, moment aigu de déliaison, mais créant paradoxalement un lien intense entre ceux qu'elle met à l'épreuve. La guerre est en embuscade sur le cours de l'Histoire, l'empêchant à jamais de se prendre pour un long fleuve tranquille, sauf à prendre à rebours l'aptitude à l'assoupissement. Bref, elle est un visage de l'Histoire même. 
Mais la guerre, objet inconscient ? La récurrence des métaphores militaires dans la métapsychologie freudienne ${ }^{5}$ le confirme. La conflictualité psychique impose cette dimension agonale, voire agonistique. Admirateur de Masséna, le maréchal napoléonien, dans sa jeunesse, Freud métapsychologue ne considère-t-il pas l'espace psychique - car «psyché : quelque chose d'étendu ${ }^{6}$ » - comme traversé de forces ? Le métapsychologue a sa cartographie d'état-major - nommée topique -, sa logistique dénommée économie et sa lecture stratégique - baptisée dynamique. Cela fait dudit métapsychologue un stratège, trouvant même son inspiration dans la peinture militaire, comme nous le verrons.

\section{Réel belliqueux et désir de paix}

La guerre, donnée historique massive, envahit l'histoire sur le mode irruptif. Le « traité de paix » n'est qu'un entre-deuxguerres. "Huit mille traités de paix », ainsi s'intitule tel écrit ${ }^{7}$ référé à la «polémologie » ou science de la guerre, objectivement ironique, car la multiplicité des « traités de paix » signe le caractère récurrent du phénomène d'affrontement. Quand la paix se met à durer, l'on peut en quelque sorte se tenir pour satisfait d'avoir battu un record de non-guerre... C'est donc, comme réel, ce qui est premier, la paix n'étant qu'une pause ou une diversion, autant qu'un contraire. Il y a une « comédie de la paix ${ }^{8}$ », ce qui en fait un thème tragicomique ; mais il $\mathrm{y}$ a bien aussi un désir de paix, d'une « paix perpétuelle » disait $\mathrm{Kant}^{9}$, qui aille au-delà des simples et multiples « cessations d'hostilité ». Idéal régulateur, tendant à remplacer une série de petites paix conjoncturelles et provisoires par la Paix proprement dite, qui n'est en effet rien de plus qu'une Idée régulant la Raison politique, Kant n'étant pas un doux rêveur, position fatale pour penser la guerre.

5. Cf. l'analyse du champ métaphorique freudien notre Dictionnaire des cuvres psychanalytiques, op. cit.

6. Aphorisme, août 1938.

7. G. Bouthoul, Huit mille traités de paix, Paris, Julliard, 1948.

8. P.-L. Assoun, «Éros et Polemos : la comédie de la paix », dans Analyses \& réflexions sur Aristophane "La Paix », Paris, Éditions Ellipses, 2002, p. 107-114.

9. E. Kant, Vers la paix perpétuelle, 1795 ; Paris, Vrin. 
Si la vie se définit de résister à la mort, avec les ressources de ses «fonctions », comme le soutient Bichat, la paix est ce qui manifeste la résistance à la guerre... sauf qu'il y a des paix de mort et des guerres dont la nécessité est vitale. Ce dont l'impératif « Faites l'amour, pas la guerre », l'adage hippie des années 1970, organise l'évitement, vœu pieux, plus facile à nourrir sous toxique. Suggestion qu'en effet face à Thanatos, seul Éros fait le poids, mais qui méconnaît qu'il arrive que le sujet, plus souvent qu'à son tour, jouisse plus de la destruction que de l'amour. Et puis des esclaves vaincus qui font l'amour sous l'œil du Maître vainqueur, cela fait une drôle d'érotique (dont seul le masochiste pourrait s'inspirer). Sans compter l'essentiel, celui de ne pas envisager que l'amour, ce puisse être la guerre, ce que démontre aisément la chronique du couple humain...

\section{De «la fleur au fusil » à la guerre lasse d'elle-même}

Pourquoi donc la guerre « éclate »-t-elle ? Pourquoi, question au moins aussi révélatrice, cesse-t-elle ? Comment la fleur peutelle orner le fusil ${ }^{10}$, ce qui fait prendre au début des guerres un aspect d'amours débutantes, de débordement de jouissance collective, comme en 14, où le lien national se fait sentir « au pas de charge »- avant de se transformer in fine en chrysanthème ? Il y a dans l'expression cette idée que c'est l'arme - celle qui donne son nom à « l'armée »-qui fait jaillir la flamme, comme du silex (« pierre à fusil ») en tant qu'outil fantasmatique de refleurissement, soulagement drastique du malaise chronique du temps de paix. Longuement redoutée, la guerre peut avoir l'effet immédiat d'alléger d'une « attente anxieuse », inversée du jour au lendemain en «attente croyante ». La paix sépare à sa façon, là où la guerre unirait, en une effusion maniaque, par désignation de l'ennemi commun. Le va-t-en-guerre « comme un seul homme » : voilà un vertige qui révèle le gain de la guerre, en son moment d'initium. C'est un fait que la guerre fait chanter, sur l'air de Malbrough s'en va-t'en guerre, rengaine irrésistible depuis

10. L'expression a trouvé son texte dans J. Galtier-Boissière, La fleur au fusil, publié en 1928 (paru en 1917 sous le titre En rase campagne 1914, version qui fut censurée). 
le XVIII ${ }^{\mathrm{e}}$ siècle, où se démontre que la dérision de l'ennemi peut contribuer à le populariser, Malbrough devenant un emblème « comico-tragique » du destin du combattant ${ }^{11}$.

Comment, par ailleurs, le combat peut-il cesser ? «Faute de combattants ${ }^{12} »$, certes, mais aussi comme limite atteinte : l'expression « de guerre lasse » pourrait être le signifiant marquant, démarquée de l'ablatif latin - « la guerre étant, devenant lasse », alors même qu'elle s'avère aussi inlassable. Car la guerre produit une fatigue d'un genre spécial, extrême, par ce qu'elle prélève sur les corps. L'expression suggère non seulement que les guerriers peuvent être profondément las de guerroyer, mais que la guerre en personne, celle que font les hommes mais qui s'avère les dominer, tel Polemos, peut devenir lasse d'elle-même, se prendre en dégoût - ce qui permet au moins une pause et un entracte et génère un goût inédit de la vie, une aspiration au bonheur difficilement supportable en temps de paix. «La » guerre, création allégorique, est faite de l'agressivité humaine, mais elle semble en prendre possession, à la façon d'un fatum, en sorte qu'elle impose sa loi et crée un état d' « anomie » où tout semble possible, introduisant une scansion dans le temps collectif.

\section{Freud à l'épreuve de la guerre}

Alors, Freud ? Lui est confronté à l'invention de la « guerre de masse ». À titre personnel, il se déclare partisan résolu de la paix, plutôt que « pacifiste ». Pas besoin même d'insister, cela va de soi, dès lors que l'on se revendique comme " homme de culture ». Krieg et Kulturmensch sont deux réalités réciproquement allergiques. Mais il y a bien des formes de pacifisme, dont la plus active, en son temps comme en tous, celle d'une lâcheté rationalisée en idéal, d'une propension à la servitude déguisée en défense de la Liberté. Que l'on pense à Munich, les 29 et 30 septembre 1938, un an avant la mort de Freud, quand l'Europe capitule face à Hitler pour sauver une paix dite déshonorante et à la filiation du pacifisme avec la collaboration, l' « effet Munich »

11. La chanson raconte les aventures de John Churchill, duc de Marlborough, allusion à la bataille de Malplaquet le 11 septembre 1709, la version française tournant en dérision le personnage, mais le succès en produisit une héroïsation. 12. P. Corneille, Le Cid, acte IV, scène 3. 
étant depuis lors récurrent en Europe où il demeure présent à l'état torpide.

De façon structurelle, rien ne répugne plus à Freud que l'idéalisme en politique. On connaît son antipathie viscérale envers Wilson ${ }^{13}$, les intentions les meilleures étant le pavé électif de l'enfer, dans l'ordre politique. Celle de Freud est fondée sur la conviction que la guerre est inhérente à l'humain, que c'est le réel même de la pulsion de destruction. Elle met au pied du mur de cette pulsion en ses effets ravageants. Freud assume à la face de tous la contradiction, chez lui-même, de ne pas céder sur le désir de paix, alors qu'on n'en trouve pas de trace dans le réel de l'inconscient, pas plus que de quelque «pulsion de perfectionnement ${ }^{14} »$. Le désir de paix est, chez Freud comme chez Kant, quoique sous des modalités différentes ${ }^{15}$, un impératif de la Raison politique. C'est pour celui qui, loin de tout irénisme, sait la prégnance de la guerre, que la paix se pose en impératif catégorique du «droit politique ». L'adage «Si tu veux la paix, prépare la guerre » (si vis pacem, para bellum) est déduit du réalisme de l'affrontement. Ceux qui méconnaissent le risque de guerre au nom d'un universel consensuel ignorent qu'ils sont désignés par l'ennemi comme tel. On en voit les effets : périr, étreignant leur fantasme de paix, sous les coups de l'ennemi réel. Si la question est datée, elle prend date de façon éloquente pour le présent qui ramène inlassablement la structure.

\section{«Pourquoi de la guerre?»}

Nous voilà donc face à la question la plus « réelaliste ». Pourquoi faut-il de la guerre à l'homme?

On notera que lorsqu'il parle de la guerre et de la mort, Freud le fait à chaque fois comme une adresse, avec un destinataire donc : de l'association Bnai'brith en 1915, association juive à laquelle il s'adresse depuis sa «traversée du désert », en parlant de la mort,

13. Voir l'étude de Freud sur Le président Thomas Woodrow Wilson. Portrait psychologique, écrit à la fin de sa vie en collaboration avec W.C. Bullitt et qui ne fut publiée qu'en 1966.

14. S. Freud, Au-delà du principe de plaisir (1920).

15. P.-L. Assoun, «Le désir perpétuel. Guerre et paix de Kant à Freud », dans «Vers la paix perpétuelle » de Kant, Paris, Éditions Ellipses, 2002, p. 161-172. 
à la Société des nations en la personne d'Einstein en 1932. Nous sommes bien dans l'élément du discours, en sa fonction sociale, celui du citoyen Freud, mais le créateur de la psychanalyse ne peut faire abstraction des enseignements anthropologiques de sa clinique de l'inconscient. On ne s'entretient pas de la guerre de soi à soi (si ce n'est dans le délire où elle peut s'avérer pour le psychotique un ennui personnel), mais en se plaçant au dehors car la guerre est un dehors, elle exige une «pensée du dehors ». Façon de se " mettre au-dessus de la mêlée », comme Romain Rolland, mais sans pathos, sachant que la guerre produit une mêlée à laquelle nul n'échappe. Même quand il parle de la Vergänglichkeit que l'approche de la guerre révèle, c'est encore un dialogue qu'il instaure, quoique plus confidentiel, voire intime de ceux qui se promènent sur les bords de l'Histoire. La guerre, cette figure de la fureur collective, incite aussi au discourir : elle est en effet ce dehors qui convoque à la parole. Notamment au titre qu'elle révèle l'irruption du vergänglich au cœur de la Culture.

\section{Le drame de la guerre}

Il apparaît à l'examen que l'effort de Freud est de décrire, avec les ressources de la psychanalyse, la situation psychique correspondant à l'état de guerre (Kriegszustand), au-delà de ses coordonnées politiques, économiques et historiques, essentielles à prendre en compte par ailleurs. Si la guerre est «mauvaise » et destructrice, elle est bien une donnée anthropologique. De plus c'est bien l'ennemi le plus implacable qui enseigne au sujet sa vérité, plus que l'ami complaisant, qui en soutient peu ou prou l'illusion. Comme pour le toxique, il ne suffit pas d'en déplorer l'existence (« il eût fallu qu'elle n'existât point »), la guerre ne s'écrit pas au subjonctif et à l'optatif, mais à l'indicatif, modalité du trauma. Un processus intervient ici, celui de la « dramatisation $^{16} \gg$. Processus que Freud postule dans le procédé du rêve : « À partir de ces images, le rêve met en forme une situation, il présente une chose comme étant au présent, il dramatise une idée. » Présentation de la pensée au présent avec omission du

16. Le terme «dramatisation » est institué dans l'essai sur Le rêve (1901), mais il est décrit comme l'action de «dramatiser » dès L'interprétation du rêve. 
«peut-être ». Il y a bien en ce sens un drame de la guerre, corrélatif du vécu de dramatisation : en entrant sur le théâtre de la guerre, le sujet se voit confronté à l'actualité de l'agressivité, ce qui lui donne ce caractère d'invraisemblable réel, dans un climat cauchemardesque.

Il s'agit de se demander pourquoi l'individu comme l'humanité donnent à la guerre « une place fixe dans leur libido »... Au-delà du discours d'indignation déploratoire, il s'agit de saisir le réel belliqueux, meilleure façon de tenter de répondre à la question «que faire ? » ou qu' « en faire ? " Envisager de se préserver de la guerre suppose d'expliquer comment ça marche ( « au pas ») et quel est cet amour obscur de la violence qui travaille l'inconscient. La question de la «prévention » (Kriegsverhütung) est bien l'enjeu de la correspondance suscitée par l'Institut de coopération intellectuelle de la SDN entre Freud et Einstein. Verhüten, c'est empêcher que survienne quelque chose d'indésirable. Le problème est qu'il y a justement un Wunsch de guerre, une " bellicité », plus radicale encore que le caractère belliqueux de circonstance.

\section{LA GUERRE OU LA VÉRITÉ DE LA MORT}

\section{De l'angoisse de l'avant-guerre à la guerre, épreuve de vérité du sujet}

Le diagnostic freudien quant à l'irruption de la guerre comporte deux éléments essentiels : désillusion par rapport aux idéaux de la Culture et modification de l'attitude envers la mort ${ }^{17}$.

La guerre, considérée dans l'absolu, est susceptible d'ouvrir les vannes de la barbarie. À ne pas confondre avec la sauvagerie, déchaînement de cruauté - dans le versant de terreur, qui, elle tend à faire surgir la guerre au cœur de l'état de paix ${ }^{18}$ : la

17. S. Freud, « Notre rapport à la mort », dans « Actuelles sur la guerre et la mort »(1915), dans Euvres complètes, volume XIII, Paris, Puf, 1988. Sur le contexte de ce texte et son contenu, cf. l'article qui y correspond dans P.-L. Assoun, Dictionnaire des æeuvres psychanalytiques, op. cit., p. 173-176.

18. P.-L. Assoun, «Le préjudice radical : de l'idéal à la destruction », dans F. Benslama (sous la direction de), L'idéal et la cruauté. Subjectivité et politique de la radicalisation, Paris, Lignes, 2015, p. 47-67. 
barbarie dénote une attitude hostile envers la Culture même. Le texte sur " l'éphémérité ${ }^{19}$ » décrit, situé rétrospectivement dans la période immédiate de l'avant-guerre, cette crise qui s'inscrit dans la subjectivité en angoisse et en mélancolie pour les idéaux de culture. Il y a bien en effet un climat spécial de «l'avant-guerre », cet état particulier d'anomie qui précède le découpage en deux de la temporalité, par l'irruption de ce météorite, où se décompose la subjectivité, avant d'exploser. État qui est comme le «phénomène élémentaire » du déclenchement guerrier et qui fait partie de plein droit du circuit de la guerre, celui du «non-encore-arrivé » ou « pas-encore-arrivé ». La guerre s'avère avoir commencé assez longtemps avant d'avoir éclaté : on peut le reconnaître à des signes cliniques précis. C'est ainsi qu'il faut relire la promenade sur le bord de l'Histoire, dans ce climat ouaté qui précède le cliquetis des armes - car la guerre est bien de l'ordre du charivari-, remémoré après que la guerre ait commencé et qui prend son sens par cet après-coup.

\section{La mort ou «l'état d'exception » au quotidien}

Mais, de plus, l'état de guerre instaure un rapport d'exception à la mort, en l'inscrivant dans la chronique du quotidien. C'est l'occasion funèbre où l'on « compte les morts », ce qui modifie le rapport à $l a$ mort. Être et vivre en état de guerre, soit comme combattant soit comme spectateur, au front ou à l'arrière, c'est être obligé de regarder la mort en face ou à tout le moins de cohabiter avec elle, les cadavres s'accumulant chaque jour.

L'enjeu en est le rapport que «nous avons à la mort », donc le « rapport à la mort » du sujet. Or le fait est que, pour Freud, celui-ci ne croit pas à sa mort, alors même qu'il sait qu'il va mourir - sauf à préciser ce qu'est dès lors un tel "savoir ». Différend non négligeable avec Lacan, pour qui l'on s'adosse à sa propre mort pour supporter les épreuves du vivant et du désirant : «Vous avez bien raison de croire que vous allez mourir, bien sûr, rappelle-t-il à ses auditeurs mortels. Ça vous soutient! Si vous n'y croyez pas, est-ce que vous pourriez supporter la vie que vous avez ? Si on n'était pas solidement appuyé sur cette certitude que

19. S. Freud, Éphémérité, 1915, dialogue fictionné situé à la veille de la guerre. 
ça finira... est-ce que vous pourriez supporter cette histoire ${ }^{20}$ ?» Il relève néanmoins que «c'est du domaine de la foi », « ce n'est qu'un acte de foi ». Freud, lui, souligne ce détournement foncier du sujet de l'idée de «sa » mort, qui n'y accorde pas foi. De quoi en effet peut « faire foi » cet irréel fatal qu'est le devenirmort ? Forte inclination à contourner l'idée de mort qui s'avère spécialement épineuse en temps de guerre. L'intempérie rendraitelle réaliste ? La guerre fait objection à - et contraste avec - ce déni chronique, c'est la conjoncture par excellence où le déni de la mort se trouve entravé ou bien impose une exacerbation du déni, qui crée une « confusion de sentiments » totale. Telle est la « leçon » de la guerre : elle «balaie nécessairement ce traitement conventionnel de la mort. La mort ne se laisse plus dénier ; on est forcé de croire en elle. Les hommes meurent effectivement ». Les hommes meurent pour de vrai, en voilà une surprise pour ceux qui sont endormis sur le mol oreiller de la paix, qui reconduit le déni de mortalité et cette tranquille « a-mortalité », assurée par le bouclier narcissique (une arme qui peut servir en toutes saisons !). À quoi fait écho le ronflement européen de l'entre-deux-guerres, auquel le fracas des armes de «la guerre annoncée » mettra une fin brutale autant que prévisible.

Ici se fait entendre en écho l'expression «On n'a qu'une vie », voix qui s'élève alors qu'elle ne se fait entendre que mezzo voce en temps de paix. Adage d'autant plus important à rappeler et entendre ${ }^{21}$ que ledit «mortel » ne croit pas « pour de bon » à la mort qui lui est promise, ligne de franchissement à l'horizon de sa vie, épée de Damoclès dont il a l'art de se distraire. La guerre est l'une de ces situations d'expérience forcée de la mort, fréquentée intensément, qui modifie la posture du sujet envers ce réel. Surtout on meurt ensemble, quoique chacun de son côté, ou l'on voit mourir les autres. C'est ce qui crée le fossé entre ceux qui ont vécu la guerre et ceux auxquels «le direct » en a été épargné. C'est aussi ce qui explique ce climat si spécial des « après-guerre », qui montre les effets libidinaux de ceux qui se réveillent de leur fantasme d'immortalité par une excitation

20. J. Lacan, conférence de Louvain, 13 octobre 1972.

21. P.-L. Assoun, « À propos de l'expression “On n'a qu'une vie” », Interfaces Psy, $\mathrm{n}^{\circ} 1,2003$, p. 12-16, repris dans Le préjudice et l'idéal. Pour une clinique sociale du trauma, Paris, Economica/Anthropos, préface de la $2^{\mathrm{e}}$ éd., 2012. 
à vivre boulimique et un appétit de rapports neufs au désir et à l'écriture. L'état de « fureur » créé par l'état de guerre y marque sa frénésie inoubliable, qui peut s'inscrire en nostalgie pour celui qui est revenu à la paix sans ne plus jamais être en paix... C'est d'autant plus notable que l'armée se définit par le contrôle et le respect inconditionnel du commandement, bref la discipline : la guerre vient introduire la fureur dans cette « foule artificielle».

À cette occasion, Freud avance une hypothèse originale sur l'origine du désir de recherche : "C'est le conflit de sentiment à l'égard à la mort de personnes aimées et pourtant en même temps étrangères et haïes qui a libéré la recherche chez les hommes. » Face au cadavre de la personne aimée, le deuil originaire générerait la pensée. Le chercheur serait un orphelin ambivalent... La recherche sent donc l'autopsie. Et le chercheur le plus vif a quelque chose d'un médecin légiste en questionnement de son rapport à la palpitation de son objet. Cela vaut pour toute mort, mais l'accumulation de l'expérience des morts pourrait faire de la guerre un chantier de recherche d'exception.

\section{«Vivre n'est pas nécessaire » : l'attrait secret de la guerre}

Plus profondément encore, risquer la vie d'une façon ou d'une autre est une condition pour exister. L'être qui ne risque jamais sa vie est un mort-vivant. «Exclure la mort des comptes de la vie » conduit à toutes les concessions, comme dit Freud en une belle formule. Avec la guerre, c'est devenu difficile, sinon impossible, on est « payé pour savoir » qu'il faut payer la note, pour renchérir sur l'image freudienne (Berechnung désigne la « facture »). Il devient flagrant, dans la tourmente de la guerre, qu'il faut « compter avec elle » - cette mort à laquelle, selon la formule de Bichat, la vie ne fait que résister ${ }^{22}$-, si bien qu'elle la met de côté ou ne la conçoit que comme une interruption de la vie, alors que c'en est le ressort négatif.

Cela révèle que «vivre n'est pas nécessaire, c'est naviguer qui est nécessaire », comme le disait Pompée à ses soldats au moment de les embarquer sur les galères, dans une mer infestée de pirates. Mais ce faisant, « la guerre enlève les sédiments de

22. X. Bichat, Recherches physiologiques sur la vie et la mort, 1802. 
culture récents et fait réapparaître en nous l'homme originaire ». Elle « désigne la mort de l'ennemi et amène à passer outre la mort des personnes aimées ». Si l'on ne trouvera certes pas d'éloge de la guerre chez Freud - c'est hors de question pour qui assume son appartenance culturelle -, il constate que la guerre s'avère élever le sentiment d'exister, donnant non une raison d'être, mais une façon de pratiquer sa peine à être, comme l'indique la clinique du «baroudeur » ou du mercenaire... Pouvoir perdre la vie rend cette vie digne d'être vécue, comme l'indique a contrario le vécu poignant des «Libérations » ou fins de guerre (« fins de partie »).

On peut mieux le comprendre en constatant la dégradation du rapport à la vie dans les états de paix de longue durée. Les conduites dites « à risque » des temps de paix, les toxicomanies, les suicides traduisent cette dégradation de la valeur d'une vie qui ne se risque pas pour de bon. «Il leur faudrait une bonne guerre ! », s'écrie alors un certain sadisme populaire des aînés, s'adressant aux « jeunes » des temps de paix, depuis leur expérience supposée de la guerre. Pourtant, les anorexiques auxquelles on adresse ce rappel sont bien elles aussi en guerre pour exister, en temps de paix déprimants, de mise en berne des idéaux et des drapeaux familiaux. Du moins se trouve pointée au passage la question de la balance entre vie et mort. On pourrait donc soupçonner que c'est ce que cherchent les sujets à «l'école de la guerre » : « Faire à la mort, dans la réalité effective et dans nos pensées, la place qui lui revient. » D'où la conclusion : «Si tu veux endurer la vie, organise-toi en vue de la mort. » On voit que la guerre donne à l'écrit de Freud de 1915, que lui-même désigne modestement dans sa correspondance comme un «papotage d'actualité ${ }^{23} »$, la portée d'une véritable méditation sur la mort, avec les ressources de la psychanalyse, sans le pathos déploratoire afférent, car c'est le thème même où il s'agit de cesser de se payer de mots...

23. Lettre de Freud à Karl Abraham du 4 mars 1915. 


\section{CLINIQUE DU SUJET DE LA GUERRE}

\section{Le trauma de guerre ou l'angoisse du double}

Le fait guerrier doit être envisagé depuis le combattant à présent, dont Freud dit en 1915 qu'il connaît mal la psychologie, façon de ne pas s'engager alors dans un vain exercice. Car il n'y a pas de psychopathologie du combattant comme tel, plutôt une mise en acte de l'inconscient par le combat! Trois ans plus tard, juste à la fin de la guerre, alors que le fracas en est à peine retombé, il présente les «psychonévroses de guerre ${ }^{24}$ ».

Or qu'y apprend-on? Que le trauma se caractérise par l'enfoncement du «pare-excitations ». On vérifie l'afflux des métaphores militaires au cœur de la métapsychologie, de la « défense » (Abwehr) au Reizschutz, qui impose l'idée d'un « front » de guerre face à l'excitation ${ }^{25}$ ennemie. L'expérience traumatique montre la désunion pulsionnelle, le trauma étant un agent désintricateur. Ce n'est pas un hasard si l'essai sur l'«au-delà du principe de plaisir » commence, dans le relevé clinique des manifestations cliniques qui en attestent, par les névroses traumatiques, éminemment dans la situation de guerre ${ }^{26}$. Freud en parle avec un enjeu : il pressent que ce sera l'occasion, pour les détracteurs de l'explication analytique, de relativiser et de dénier la portée du trauma sexuel, en donnant à nouveau la primauté au trauma mécanique sur le trauma psycho-sexuel. Ce qui s'est confirmé depuis avec la prévalence de la théorie du «stress post-traumatique », nouvelle vulgate de la vieille théorie du « choc », dont le succès est dès lors assuré. Il convient de montrer comment le trauma d'une violence mécanique effectivement exceptionnelle est aussi la cause occasionnelle de la réactivation de traumas anciens et singuliers. Freud fait notamment une suggestion décisive sur le clivage entre le «moi de paix » et le «moi de guerre du soldat» créé par la situation de guerre ${ }^{27}$. Avancée clinique à nos yeux

24. S. Freud, Introduction à Sur la psychanalyse des psychonévroses de guerre, $\mathrm{V}^{\mathrm{e}}$ Congrès de psychanalyse de Budapest, 1918.

25. P.-L. Assoun, L'excitation et ses destins inconscients. Court traité psychanalytique de l'excitation, Paris, Puf, 2013.

26. S. Freud, Au-delà du principe de plaisir, 1920, sect. I.

27. Suggestion émise en référence à Karl Abraham. 
capitale : le sujet en guerre «tombe malade » quand il est pris en sandwich entre l'ennemi - de la tranchée d'en face - et l'ennemi qu'il devient au même instant pour lui-même, sous la pression de la situation nouvelle et dans « l'entonnoir temporel » créé par la situation inédite. Selon la formule freudienne des plus précises, " l'effondrement se produit quand devient évident au moi de paix le danger qu'il court de perdre la vie en raison des audaces de son double parasitaire nouvellement formé ». Il s'agit d'un clivage induit par le trauma envers ce dont il se ressent capable, ses Wagnisse, ses initiatives intempestives (on y entend le verbe wagen, oser), moment d'affolement de la pulsion d'auto-conservation, narcotisée par l'épreuve du feu, où il se ressent capable d'à peu près tout. Les « orages d'acier ${ }^{28}$ » déclenchent certains orages secrètement désirés, selon la formule de Chateaubriand ${ }^{29}$, quoique le dénouement n'en ait rien de romantique.

D'où la fuite dans la «névrose traumatique ». Le signe principal de la psychonévrose de guerre est un tremblement d'une violence indescriptible (on peut en juger sur les impressionnants documents cinématographiques des hôpitaux militaires de l'époque). Ce n'est pas que le sujet soit lâche - comme Freud le rappelle quand il se trouve consulté sur la question des « simulations » à la suite de l'affaire Wagner-Jauregg, le psychiatre accusé d'abus du traitement électrique ${ }^{30}-$, s'il a peur de son ombre, c'est que se trouve actualisée une tendance suicidaire compulsionnelle, terreur du sujet envers lui-même, telle que rien ne l'arrête, au point de se précipiter vers la mort à son corps défendant.

\section{De la guerre comme magie noire}

On voit la complexité du rapport à sa propre mort pour le combattant : d'un côté, il ne peut plus organiser le régime commun de déni, de l'autre, fréquentant la mort, il instaure avec elle un rapport spécial, magique, par où la guerre prend sa

28. E. Jünger, Orages d'acier. Souvenirs du front de France (1914-1918), 1920.

29. Chateaubriand, René, 1802.

30. S. Freud, « Rapport d'expertise sur le traitement électrique des névroses de guerre »(1920), dans CEuvres complètes, vol. XV, Paris, Puf, 2002. Cf.

K. Eissler, Freud sur le front des névroses de guerre, Paris, Puf, 1992. 
dimension de «magie noire ${ }^{31} »$. Le combattant doit vivre avec la possibilité chronique de sa mort - chaque jour ouvrant la question de savoir si «c'est pour aujourd'hui », chaque moment contient la possibilité de la mauvaise rencontre. Mais son double - « ange gardien », garde du corps et ange de mort - a pour fonction d'instaurer ce rapport magique qui combine reconnaissance et déni du danger mortel. Cela permet de vivre en temps de mort...

On le vérifie à l'aura de tabou qui entoure celui que l'on appelle «l'ancien combattant». Expression fortement contradictoire : car « le combattant» est l'homme du moment, il s'écrit au " gérondif», soit celui qui est en train de combattre. L' " ancien» combattant est celui qui s'écrit au passé, marquant le retour du refoulé - comme le «mutilé de guerre » qui, passé le moment de l'héroïsme du martyr, vient angoisser le train-train quotidien. Celui qui « en est revenu » notifie que son double l'aura bien conseillé ou épargné, le plaçant au bon endroit où l'obus ou autre projectile ne l'aura pas frappé, « de justesse » comme on dit. Drôle d'expression décidément, car le « combattant» est par définition en acte : c'est aussi pourquoi lesdits « anciens » forment des associations et ont tant de mal à vieillir : survivre en état de paix, c'est un défi redoutable pour ceux dont le corps reste, telle une aiguille, fixé à l'heure de la guerre et de la mort imminente. «Que me veut l'Autre pour m'avoir épargné ? », la question rôde autour de telles problématiques subjectives.

« Magie noire », donc, mais qui montre qu' au cœur du bruit et de la fureur de la guerre, le « Maître absolu » qu'est la mort n'est pas affronté de façon frontale, même au bien ou mal dit « front ». Il faut là encore savoir jouer (« au plus fin ») avec le Maître - ce qui produit cette variante d'humour noir qui questionnerait le surmoi ${ }^{32} \mathrm{du}$ combattant (et qui en assure la fascination littéraire et cinématographique). C'est de perdre le combat contre son double que le sujet prend le chemin de «l'hôpital militaire ».

31. P.-L. Assoun, « Magie et psychose. La magie à l'épreuve de la psychanalyse », Cliniques méditerranéennes, ${ }^{\circ}$ 85, 2012, p. 59-76.

32. Sur le lien entre surmoi et humour, développé dans l'essai de Freud sur L'Humour, cf. nos contributions « Le sujet de l'humour ou le surmoi humoriste », dans La matière et l'esprit, $\mathrm{n}^{\circ}$ 24, « Humour et identité », 2012, p. 25-52 et «Le moment ou jamais : le sujet de l'humour», Champ Psy, ${ }^{\circ} 67$, «L'humour et le rire », 2015, p. 25-46. 
Le «front » et «l'arrière » :

acteurs et spectateurs de la guerre

La guerre découpe le monde social et ce que l'on appelle « nation » en deux portions : le « front » et « l'arrière » qui, en allemand, se dit Hinterland, " le pays de derrière ». La guerre vue respectivement côté militaire et côté civil, en sorte qu'elle n'est pas la même. Freud, ne le perdons pas de vue, parle depuis le spectateur, "non mobilisé ». Le non-combattant, volontiers présenté comme "féminisé », est celui pour qui la guerre est réduite à un regard. Ce qui n'est pas toujours une sinécure : ainsi s'ouvre la pathologie du spectateur. S'il n'est directement confronté à l'épreuve du sang, il est exposé à la passivation de celui qui remplit son regard du trauma de et dans l'autre.

Car la guerre est bien une figure du spectaculaire. Le philosophe-soldat Gray en donne une description lucide, en la situant aux confins de la « jouissance de voir » et de la « jouissance de destruction », avec, au centre, l'aptitude à créer du fraternel par le feu du combat et l'exposition, un par un, au même danger. Étrange érotisme de déplacement des " masses d'hommes », ce qui en fait un chapitre de la Massenpsychologie : « Il y a souvent une étrange mais authentique beauté, constate Gray, dans le spectacle d'hommes et d'armements massés au combat ${ }^{33}$. » Pour revenir à l'affect militaire dont nous sommes partis, on dit que c'est « le spectacle de colonnes avançant sous le feu ennemi » qui aurait arraché à Lee, pendant la guerre de Sécession, cet aveu d'une beauté de la terreur. Mieux : le rappel à soi de la terreur fait barrage, au cœur de la guerre, à la jouissance du spectacle destructif, mais ne la voile que très relativement. Ainsi de la fascination des armées alliées, évoquées par Gray, prêtes à débarquer, contemplant le ciel illuminé par les avions, feu d'artifice libérateur.

Mais d'un autre point de vue - c'est le cas de le dire -, de la guerre elle-même, on ne voit rien. Ce qu'expérimente le Fabrice stendhalien à Waterloo, essayant de passer de l'état de spectateur à celui d'acteur et faisant l'expérience de ne rien voir, restant sur le bord du champ de bataille - ce qui ne lui en vaut pas moins une

33. G. Gray, Au combat, op. cit., p. 75. 
réputation de héros auprès des dames ${ }^{34}$. Mais on sait aussi que, comme dans les traumas familiaux, c'est celui qui assiste aux sévices qui se trouve souvent le plus «malade ». Par un retournement de la passivité à l'activité (et non, comme dans le destin pulsionnel, de l'activité à la passivité), il peut en arriver à faire couler le sang dans l'après-coup, pour s'approprier, en un fading, la scène de violence dont il fut originairement exclu comme acteur. Ainsi s'enkyste la jouissance. De façon plus (faussement) tranquille, celui qui demeure à l'arrière, réputé «planqué » à l'occasion, assistant à la mort de l'autre, organise un rapport de culpabilité passivante mais fantasmatiquement active, qui fait que c'est lui, paradoxalement, qui peut se coltiner un rapport au réel de la mort, que l'homme du front aura su déjouer par les pratiques magiques évoquées... Nous sommes là au cœur d'une clinique du sujet en guerre.

La veine militaire de la sorcière métapsychologie nous fait signe à nouveau : sous la plume du métapsychologue, la comparaison s'impose pour désigner la place respective du «moi » et du «ça » : «Pensez à la différence du front et de l'arrière, tel qu'il s'était formé pendant une guerre. Nous ne nous sommes jadis pas étonnés qu'au front beaucoup de choses se passent autrement qu'à l'arrière, et qu'à l'arrière beaucoup de choses sont permises, qui devaient être interdites au front. L'influence déterminante était naturellement la proximité de l'ennemi, pour la vie psychique, il s'agit de la proximité du monde extérieur. Dehors - étranger - ennemi ne sont que les mêmes concepts ${ }^{35}$. » Ainsi, le «ça » est l'arrière et le «moi » le front. Par ailleurs, dessinant le champ de combat des instances, le moi, instance de la perception, affronte - frontalement - le monde extérieur, il doit même se battre de plus contre les deux fronts, du ça et du surmoi ${ }^{36}$, tandis que le ça ignore les conflits. On aurait pu penser que le ça du combattant puisse trouver à se déchaîner à la guerre, mais Freud, en ce contexte, présente l'instance pulsionnelle comme protégée du conflit. Cela se confirme par un certain visage de l'arrière comme lieu de débauche...

34. P.-L. Assoun, «Imaginaire héroïque et féminité. Psychanalyse de "La Chartreuse de Parme" ", dans collectif, Analyses et réflexions sur Stendhal. La Chartreuse de Parme, Paris, Ellipses, 2000, p. 94-104.

35. S. Freud, La question de l'analyse profane, sect. II, G.W. XIV, p. 223.

36. S. Freud, Abrégé de psychanalyse, G.W. XVII, p. 120. 


\section{La «pulsion de destruction » en acte}

Notons bien que «Pourquoi (de) la guerre ? » commence par un long cours de géopolitique. Freud va même jusqu'à suggérer à son correspondant Einstein que lui, le physicien, a dit l'essentiel au plan " psychologique », une fois qu'il a pris acte de cette pulsion destructrice. C'est une fois achevé cet état des lieux « politicologique » qu'il introduit ce que la psychanalyse a de plus spécifique à dire, cette pulsion destructive et mortifère comme dérivée de la pulsion de mort, qui va bien au-delà d'une vague inclination à la violence. Façon de signifier que mettre l'accent sur la tendance à l'agressivité n'est qu'un lieu commun abstrait (comme tout lieu commun), tant que, de cette pulsion, l'on n'a pas nommé le vrai ressort métapsychologique, la pulsion de mort, à travers son débouché destructeur. La guerre est l'un des états qui permet de prendre la mesure de cette " mise au dehors » de la pulsion de mort, dont elle est la forme pratique et, en un sens, le masque. Là vient à la rescousse la pensée présocratique d'Empédocle d'Agrigente ${ }^{37}$ qui parle du Neikos, ce que l'on restitue comme « Haine », mais qui désigne plus littéralement la «Dispute », combat en mots et en actes, ou encore la « Discorde ». Elle s'oppose en ce sens à la Philia, l'Amitié ou Concorde, ce qui donne de faux airs de «Champs catalauniques ${ }^{38}$ » aux guerres de tous les temps. Cette multiplication d'entités majuscules ne doit pas faire perdre de vue qu'elles sont à retraduire métapsychologiquement en termes de forces pulsionnelles, mythes à « la grandiose indétermination ». Est-ce un hasard si Arès (Mars), le dieu de la guerre, est marié à Aphrodite (Vénus), donnant naissance, dans l'une de ses généalogies, à un certain Éros ? De son père, Éros conserve la nature belliqueuse.

Pour soupçonner qu'il y a une pulsion de destruction, il n'y a pas besoin de la psychanalyse, ce qu'elle apporte en propre, c'est la dérivation de cette Destruktionstrieb de la pulsion de mort en

37. S. Freud, L'analyse infinie et l'analyse sans fin.

38. Cette journée, qui en juin 451 décida de l'arrêt des Huns d'Attila par les armées gallo-romaines et germaniques dirigées par Aetius, inspira la comparaison de Freud dans Le moi et le ça, d'une lutte qui se poursuit au niveau plus élevé du surmoi, comme dans le tableau éponyme de Wilhelm von Kaulbach, Die Huhnenschlacht. 
toute sa portée clinique, ainsi que sa tension avec les pulsions de vie, cœur de la violence interne ${ }^{39}$. Ce « discord» majeur qu'est la guerre réalise la situation de plein-emploi... de la pulsion de mort ${ }^{40}$ en son plein empan de destruction, n'étant supplanté que par la terreur ${ }^{41}$, qui fait la guerre aux civils et dont on aura soin de la distinguer. Le terme Todestrieb figure dans le texte sur la guerre, mais il est nettement supplanté par celui de «pulsion de destruction »(Destruktionstrieb). La pulsion de mort, expression de cette tendance fondamentale du vivant et de la vie psychique à revenir à un état antérieur, inorganique, se manifeste par la répétition de conduites qui échappent au contrôle du principe de plaisir du vivant. La pulsion de destruction, qui trouve son usage guerrier, n'est pas originaire, il n'y a pas chez Freud de « violence fondamentale » (à part celle du père de horde, cet être violent et intolérant !). C'est donc bien " une dérivée » de la « fonction» pulsion de mort. C'est aussi bien un effort de la libido pour « lier» la pulsion de destruction. C'est pourquoi les «bêtes de guerre », comme d'autres acteurs d'une violence radicale, cherchent dans la situation d'affrontement une façon de porter à l'expression et de drainer la pulsion de mort interne. En témoignent les « viols de guerre » : actes où la violence destructrice s'administre par l'arme de l'acte sexuel, l'Éros montrant à cette occasion sa face de destruction, sous l'effet de la désintrication. La régression trouve son explication métapsychologique, comme le souligne Freud, dans la désintrication pulsionnelle, celle qui ramène la libido à sa phase sadique $^{42}$. Le viol collectif est donc bien le phénomène où se trahit sinistrement cette coalescence déliaisive entre sexualité et destructivité.

La haine se confirme comme ce qui « montre le chemin aux pulsions de morts ». On le voit chez Hitler qui, une fois qu'il a trouvé l'assiette de sa haine dans la rencontre du «Juif » dans

39. Il est curieux de voir un penseur aussi attentif que Gray, côtoyant de près la théorie freudienne qu'il cite, accréditer brusquement le lieu commun d'une supposée réduction de l'explication psychanalytique pulsionnelle à une dimension d'« animalité »!

40. P.-L. Assoun, « Guerre et paix selon Freud. Destins collectifs de la pulsion de mort », Topique $\mathrm{n}^{\circ}$ 102, 2008.

41. P.-L. Assoun, «Le préjudice radical : de l'idéal à la destruction », op. cit.

42. S. Freud, Inhibition, symptôme et angoisse. 
les rues de Vienne, trouve l'assurance effroyable qui va vers la «solution» de destruction totale. La haine pure cherche sa « solution » terminale. Être sûr de sa haine, cela donne une volonté d'enfer. Suggestion d'une actualité chronique : de tels sujets se refont une santé par la « haine pure », " panneau indicateur » (Wegweiser) des pulsions de $\operatorname{mort}^{43}$. On en reconnaît des exemplaires à chaque moment de l'histoire. " Pourquoi tant de haine ? », cette question récurrente et désespérément naïve s'éclaire ainsi par la réponse de l'inconscient : pour nourrir la pulsion de destruction et acter la pulsion de mort. Le béat étonnement face aux accès de haine extrême traduit la méconnaissance de la Todestrieb sous toutes ses formes, celle que nous reconnaissons, quoiqu'elle nous étonne par sa capacité au pire.

\section{«Tomber au combat» : l'objet de la chute}

Cela permet d'entendre l'expression «tomber au combat»: le guerrier trouve la mort en « tombant au champ d'honneur », plutôt qu'il ne meurt. Contraste avec la «mort civile », avec ses interminables soins palliatifs. On parle bien en espagnol des caidos, par où il faut entendre ceux qui ont fait une chute dans la mort, après un trajet « à tombeau ouvert », par où ils se sont héroïsés. Identification à « l'objet $a$ », démontré dans la mort mélancolique, qui fait partie du destin de guerre. Le corps combattant, qui chute dans la mort, définit un champ de gravitation, à l'endroit même qu'il localise de sa dépouille mortelle.

Mais c'est, pour le corps en guerre, au prix d'affronter l'épreuve du double. Il est essentiel de s'aviser que les états de guerre, sous toutes leurs formes, créent les conditions de cette mise en acte du clivage et de la décharge de l'agressivité envers soi autant qu'envers les autres, ou plus précisément de "désintrication » des pulsions de vie et de mort. Elle crée la conjoncture explosive de la rencontre entre la peur de l'ennemi et l'angoisse envers soi, occasion, pour les "mystérieuses tendances masochistes du moi ${ }^{44} »$, de battre leur plein. Point de vue autrement plus profond et réaliste que le standard des « syndromes post-traumatiques ».

43. S. Freud, Le moi et le ça.

44. S. Freud, Au-delà du principe de plaisir, G.W. XIII, p. 11. 
Cet objet, déchet héroïque, réalise, par l'événement de son extraction, l'avènement du puissant fantasme guerrier.

\section{Du Malaise des temps de paix à l'état de guerre}

Ramener «les horreurs de la guerre » à la pulsion de mort, c'est révéler, en amont, le trajet de l'Unbehagen - " malaise » qui est le destin « pacifique » des pulsions de mort - au Krieg, la déclaration de guerre ouverte, en aval. Cette généalogie est essentielle, pour ne pas démoniser la guerre, en lui faisant porter tout le poids de Thanatos et comme surgissant de nulle part, tel un coup de tonnerre dans un ciel serein, alors qu'elle en est ni plus ni moins que l'acmé, le déclenchement belliciste.

La même pulsion de mort œuvre sous ces deux formes antithétiques dans les deux états. C'est en ce sens que Malaise dans la culture et Pourquoi la guerre? sont deux écrits qui se font miroir, en une proximité plus que chronologique. Cela se trahit au détail de l'analyse formelle comparée de la structure argumentative des deux écrits ${ }^{45}$, à savoir le surgissement, abrupt et nécessaire, de la pulsion de mort au milieu, au mitan de chacun des deux écrits, comme ce sans quoi ni le malaise des temps de paix ni l'événement de la guerre (créant ce temps particulier, comme isolé, de l'affrontement) ne sont déchiffrables. Là où le malaise se maintient sans cesse en deçà du symptôme, comme un état torpide d'agressivité rentrée, la guerre a la franchise d'une crise qui vient au grand jour. Moment où « la violence marche le front haut », selon la forte formule kantienne ${ }^{46}$. La pulsion de mort se révèle dans la ligne de fracture qui s'inscrit dans le discours analytique même.

\section{L'idéal à l'épreuve du feu : le phallus}

Point de guerre - faut-il le rappeler ? - sans Armée. Or, l'armée, cette « foule conventionnelle », fonctionne à l'idéal. Elle réalise bien une fabrique d'idéaux.

45. Cf. P.-L. Assoun, Dictionnaire des auvres psychanalytiques, op. cit., p. 757 et 959 .

46. E. Kant, Doctrine du droit. Cf notre commentaire dans Tuer le mort. Le désir révolutionnaire, Paris, Puf, 2015. 
L'acteur et l'organe de la guerre, c'est bien cette institution, née de ce que les individus font somme de mettre un seul et même objet au lieu de leur idéal du moi et de s'identifier les uns aux autres, dans leur moi, via cet idéal ${ }^{47}$. Cet idéal du moi, c'est l'héritier et la reviviscence de ce père mort et "résurrectionné ». Le métier militaire se situe aux confins de l'idéal (qui unit) et de la mort infligée. Faire la guerre suppose l'uniformatisation $\mathrm{du}$ « marcher au pas » et du «marquer le pas », automatisme de « marionnette » qui traduit la rigidité nécessaire sans laquelle il n'y a pas de discipline des armées. C'est aussi ce qui produit cette identification (des «moi » entre eux), dont le mot « fraternité » est la version sublimée.

Idéal à l'épreuve du feu. L'arme forgée par Héphaïstos, le forgeron mythique, qui est aussi celui des volcans, qui crachent le feu. La guerre est une activité volcanique, qui déverse des laves sur une terre ainsi volcanisée. Dans le texte où il dégage l'origine du feu, Freud le connecte à l'excitation et au feu intérieur du phallus ${ }^{48}$. Phallus en parade, en l'occasion armé et galonné. Freud n'hésite pas par ailleurs, en critiquant l'attitude des officiers allemands envers leur propre armée, à évoquer l'idée d'une machine phallique, en l'occurrence endommagée par ses propres usagers, les officiers.

\section{Les leçons freudiennes de la guerre}

La contribution psychanalytique à la question de la guerre, qui se révèle un objet essentiel pour « l'anthropologie psychanalytique $^{49}$ », est récapitulable comme moment de vérité d'une version du sujet, resté latent à l'état de paix.

En premier lieu, la guerre, qu'elle soit injuste ou produit d'une nécessité politique (ce qui, sur le plan politique, suppose de ne pas mettre toutes les guerres «dans le même sac » comme un grand malheur du monde), revient à un ravage pour les idéaux de la Culture : «l'homme de culture » est en deuil pendant la guerre.

47. S. Freud, Psychologie des masses et analyse du moi (1921), ch. VIII et notre commentaire dans Freud et les sciences sociales. Psychanalyse et théorie de la culture, Paris, Armand Colin, 2008.

48. S. Freud, Sur l'acquisition du feu.

49. P.-L. Assoun, Freud et les sciences sociales, op. cit. 
C'est pourquoi Freud constate qu'il est paradoxal d'être pacifiste pour ceux, tel lui-même (ou Einstein), qui se placent dans l'ordre du savoir, alors que la guerre est « conforme à la nature, biologiquement bien fondée, pratiquement à peine vitale ». Mais « pour des raisons organiques » constitutionnelles, l'homme de Culture, par sa « constitution », ne peut l'admettre sans se démentir. Cela traduit au reste une sorte de «division » en Freud qui, comme clinicien, trouve la guerre logique et, comme homme de Culture, inadmissible. La condamner donc sine ira et studio, sans la prothèse de l'indignation, qui vient toujours « après la bataille »...

En second lieu, c'est la rencontre du réel. L'état de paix végète dans la réalité, au moyen du déni, l'état de guerre met le sujet sur le front du réel, dont la réalité, au dire de Lacan, n'est que « la grimace ». Le nom de ce réel, c'est le trauma, cette petite mort qui déchire le voile de la vie et y grave ses cicatrices. De la clinique du combattant ou de "l'ancien combattant », qui demeure enfermé dans sa jouissance, du "mutilé de guerre », mais aussi du spectateur, celui dit « de l'arrière », spectateur souffrant, impuissant et honteux, mais à l'occasion jouissant : sauf à vérifier que c'est celui qui assiste à la violence qui localise l'épicentre de l'événement traumatique. Elle a une fonction de « démasquage ». Cela renvoie à toutes les dimensions de guerre, pendant l'état de paix, où la « violence verbale » devient l'organe de la haine rentrée, le discours de haine préparant l'acte destructif en lui donnant plus qu'une justification, un corps (discursif) de jouissance ${ }^{50}$. On soulignera ce paradoxe que le combattant ne s'endure au combat qu'en reconduisant l'élision magique du risque de mort (par le « dédoublement»), tandis que le spectateur lucide - celui que Freud instaure - est forcé à la reconnaissance du devoir-mourir.

En troisième lieu, et c'est sans doute le point le plus aigu, l'état-de-guerre permet de rencontrer la vérité du vivant, soit la mort, l'angoisse de mort n'étant pour Freud que le masque de l'angoisse de castration avec son relais surmoïque ${ }^{51}$. La guerre surmobilise le travail de résistance vitale, mais sous l'effet de la

50. P.-L. Assoun, « La haine critique ou l'envers inconscient de la violence », dans E. Marty, J. Majorel (sous la direction de), Critique et violence, Paris, Hermann, 2014, p. 6-20.

51. X. Bichat, Réflexions physiologiques sur la vie et la mort (1802). 
pression de la mort. La paix a sa façon de tuer (à petit feu) et il est avéré que quand "l'ordre règne », pour le sujet, à l'échelle de sa «petite histoire », il n'y a plus place, en cette " mort lente » reconduite de jour en jour, pour le désir, qui se déclare en revanche en une atmosphère de "déclaration de guerre ». C'est, dit au passage, toute une clinique du couple en guerre qui serait à faire, c'est certes un autre sujet, traité ailleurs, mais dont la mise en écho est saisissante si on l'aborde par la « foule à deux ${ }^{52} » \ldots$ Quand Freud évoque le témoignage de la psychanalyse que " presque toute relation de sentiment intime de quelque durée entre deux personnes [...] laisse un sédiment de sentiments de rejet et inamicaux ${ }^{53} »$, c'est la relation conjugale qu'il cite en premier, où la « guerre de tranchées » de longue durée est avérée ! La guerre proprement dite est en quelque sorte une relation intimement agressive, elle vidange cette potentialité d'inimitié en la mettant franchement en acte. Telle est la « franchise » cynique de la guerre. Reste que, comme moment collectif d'en découdre, elle en est le moment de plein-emploi... des pulsions de mort. La psychanalyse renvoie dos à dos l'imaginaire de la guerre, faisant mousser en en exaltant les vertus la jouissance et le déni idéaliste de ce qui, dans le sujet, convoite la guerre. Voie étroite, dont le savoir de l'inconscient dégage du moins l'espace : là où la pulsion de guerre continue d'être, le désir de paix insiste à advenir...

52. P.-L. Assoun, Le couple inconscient. Amour freudien et passion postcourtoise (1992), Paris, Economica, $3^{\mathrm{e}}$ éd., 2014.

53. S. Freud, Psychologie des masses et analyse du moi, ch. VI. 
